\title{
Convergence of modified homotopy perturbation method for Fredholm-Volterra integro-differential equation of order $m$
}

\author{
Zainidin K. Eshkuvatov a, d, ${ }^{*}$, Fatimah Samihah Zulkarnain ${ }^{\mathrm{b}}$, Zahriddin Muminov ${ }^{\mathrm{c}}$, Nik Mohd Asri \\ Nik Long b, d
}

a Faculty of Science and Technology, Universiti Sains Islam Malaysia (USIM), Negeri Sembilan, Malaysia

b Department of Mathematics, Faculty of Science, Universiti Putra Malaysia (UPM), Selangor, Malaysia

c Nilai University, Bandar Baru Nilai, Negeri Sembilan, Malaysia

${ }^{d}$ Institute for Mathematical Research (INSPEM), Universiti Putra Malaysia (UPM), Selangor, Malaysia

* Corresponding author: zainidin@usim.edu.my

\section{Article history}

Received 6 November 2017

Accepted 8 November 2017

\begin{abstract}
In this paper, modified homotopy perturbation method (MHPM) is applied to solve the general Fredholm-Volterra integro-differential equations (FV-IDEs) of order $m$ with initial conditions. Selective functions and unknown parameters allowed us to obtain two step iterations. It is found that MHPM is a semi-analytical method for FV-IDEs and could avoid complex computations. Numerical examples are given to show the efficiency and reliability of the method. Proof of the convergence of the proposed method is also given.
\end{abstract}

Keywords: Integral equation, homotopy perturbation method, numerical method

\section{INTRODUCTION}

Homotopy perturbation method (HPM) [1-2] is the combination of two methods: the homotopy and perturbation method. In recent years, the application of HPM in linear and nonlinear integral and integrodifferential equations arise in various fields of science and engineering has been conducted by many researchers. This method reformulated problem to a simple problem which is easy to solve. HPM has been used for a wide range of problems; for finding the exact and approximate solutions of nonlinear ordinary differential equations (ODEs) [3], one-dimensional non-homogeneous partial differential equations with a variable coefficient [4], non-homogeneous partial differential equation and fractional differential equation with initial conditions [5-6] respectively, non-linear Volterra-Fredholm integral equations and nonlinear integro-differential equations [7-8] respectively, Fredholm-Volterra integral equations and FredholmVolterra integro-differential equations (FVIDEs) of the third kind are solved in [9].

There are few modifications on HPM, one of them is adding unknown parameters to the homotopy function and define the unknown parameter by equating second iteration to be zero, it leads to two step semi-analytical method [10]. Second modification of HPM is adding a few accelerating components and selective functions to the initial approximation and find unknown paprametres by equating second iteration to be zero. It leads semianalitical solution and in many cases approches to the exact solution [11]. In 2009 [12], has introduced efficient modification of HPM that facilitates the calculations. Then, a comparative study between the new modified HPM and HPM were carried out. The modified method accelerates the rapid convergence of the series solution and reduces the size of work. Numerical examples are investigated to show the features of the technique. Another modification is used to divide the interval into subintervals and HPM is applied in each subinterval which is named multistage HPM, [13-14]. Modified HPM proposed in [11] is succesfully implimented in [15-16] for hypersingular integral equations of the first and second kind respectively.

There are many methods other than HPM to solve linear and nonlinear Fredholm and Volerra type integral equations [17-22] and integro-differential equations [23-25]. For instance, collocation method [17], the Galerkin Method [18], the iterative method [19], the moving least squares method [20], the Taylor expansion method [21] and the modified decomposition method [22]. Fredholm-Volterra integrodifferential equations arise from parabolic boundary value problems [26], the mathematical modelling of spatio-temporal development of an epidemic and various physical and biological problems [27].

In this note, the main objective is to implement modified HPM (MHPM) for general Fredholm-Volterra integro-differential equation of order $m$ with initial conditions.

$$
\begin{aligned}
& \sum_{k=0}^{m} s_{k}(x) u^{(k)}(x)=f(x)+\lambda_{1} \int_{a}^{b} \sum_{i=0}^{p} K_{1 i}(x, t) u^{(i)}(t) d t+\lambda_{2} \int_{a}^{x} \sum_{j=0}^{q} K_{2 j}(x, t) u^{(j)}(t) d t \\
& u^{(k)}(a)=d_{k}, \quad 0 \leq k \leq m-1, \quad x \in[a, b],
\end{aligned}
$$

where $s_{k}(x), k=0,1, \ldots, m, \quad p, q \leq m$ and $f(x)$ are continuous function on $[a, b], m$ is the order of differential, $\lambda_{1}$ and $\lambda_{2}$ are parameters, $K_{1 i}$ and $K_{2 j}$ are square integrable kernels and $u(x)$ is the unknown function to be determined. 
Solving Eq. (1) for $u^{(m-l)}$ leads to

$$
\begin{gathered}
u^{(m-l)}(x)=-\sum_{k=0}^{m-l-1} \frac{s_{k}(x)}{s_{m-l}(x)} u^{(k)}(x)+\frac{f(x)}{s_{m-l}(x)}+\frac{\lambda_{1}}{s_{m-l}(x)} \int_{a}^{b} \sum_{i=0}^{p} K_{1 i}(x, t) u^{(i)}(t) d t \\
+\frac{\lambda_{2}}{s_{m-l}(x)} \int_{a}^{x} \sum_{j=0}^{q} K_{2 j}(x, t) u^{(j)}(t) d t, l=\{0, \ldots, m-1\} .
\end{gathered}
$$

It is not difficult to show that if differential operator $L_{l} u$ is given as $L_{l} u=u^{(m-l)}(x)$ then the inverse $L_{l}^{-1}$ has the form

$$
L_{l}^{-1}(\cdot)=\int_{a}^{x} \int_{a}^{x_{1}} \ldots \int_{a}^{x_{m-l-1}}(\cdot) d x_{m-l} d x_{m-l-1} \ldots d x_{1}, l=0, \ldots, m-1
$$

Multiple integral of the form (3) can be written as (Kanwal [32, Appendix A1])

$$
L_{l}^{-1}=\frac{1}{(m-l-1) !} \int_{a}^{x}(x-t)^{m-l-1}(\cdot) d t
$$

Applying (4) to Eq. (2) and taking into account initial condition we obtain

$$
\begin{aligned}
u_{l}(x) & =\sum_{k=0}^{m-l-1} \frac{(x-a)^{k}}{k !} u^{(l+k)}(a) \\
& +\frac{1}{(m-l-1) !} \int_{a}^{x}(x-t)^{m-l-1}\left(-\sum_{k=0}^{m-l-1} \frac{s_{k}(t)}{s_{m-l}(t)} u^{(k)}(t)+\frac{f(t)}{s_{m-l}(t)}\right. \\
& \left.+\frac{\lambda_{1}}{s_{m-l}(t)} \int_{a}^{b} \sum_{i=0}^{p} K_{1 i}(t, \tau) u^{(i)}(\tau) d t+\frac{\lambda_{2}}{s_{m-l}(t)} \int_{a}^{t} \sum_{j=0}^{q} K_{2 j}(t, \tau) u^{(j)}(\tau) d \tau\right) d t .
\end{aligned}
$$

Operator form of Eq. (5) is

$$
u_{l}=h_{l}+L_{l}^{-1}\left\{-S_{l} u+f_{l}+K_{l} u\right\} \text {, }
$$

where, $L_{l}^{-1}$ is the inverse of differential operator defined by (4) and $K_{l}$ is integral operator of the form

$$
\begin{aligned}
& h_{l}(x)=\sum_{k=0}^{m-l-1} \frac{(x-a)^{k}}{k !} u^{k}(a), f_{l}(x)=\frac{f(x)}{s_{m-l}(x)}, S_{l} u(x)=\sum_{k=0}^{m-l-1} \frac{s_{k}(x)}{s_{m-l}(x)} u^{(k)}(x), \\
& K_{l} u(x)=\frac{\lambda_{1}}{s_{m-l}(x)} \int_{a}^{b} \sum_{i=0}^{p} K_{1 i}(x, t) u^{(i)}(t) d t+\frac{\lambda_{2}}{s_{m-l}(x)} \int_{a}^{x} \sum_{j=0}^{q} K_{2 j}(x, t) u^{(j)}(t) d t,
\end{aligned}
$$

When $l=0$, we have

$$
\begin{aligned}
u(x) & =\sum_{k=0}^{m-1} \frac{(x-a)^{k}}{k !} u^{(k)}(a) \\
& +\frac{1}{(m-1) !} \int_{a}^{x}(x-t)^{m-1}\left(-\sum_{k=0}^{m-1} \frac{s_{k}(t)}{s_{m}(t)} u^{(k)}(t)+\frac{f(t)}{s_{m}(t)}\right. \\
& \left.+\frac{\lambda_{1}}{s_{m}(t)} \int_{a}^{b} \sum_{i=0}^{p} K_{1 i}(t, \tau) u^{(i)}(\tau) d \tau+\frac{\lambda_{2}}{s_{m}(t)} \int_{a}^{t} \sum_{j=0}^{q} K_{2 j}(t, \tau) u^{(j)}(\tau) d \tau\right) d t .
\end{aligned}
$$

Writing Eq. (8) in the operator form, we get

$$
u=h+L^{-1}\left\{-S u+f_{0}+K u\right\}
$$

where

$L u=u^{(m)}(x), h(x)=\sum_{k=0}^{m-1} \frac{(x-a)^{k}}{k !} u^{(k)}(a), f_{0}(x)=\frac{f(x)}{s_{m}(x)}, S u(x)=\sum_{k=0}^{m-1} \frac{s_{k}(x)}{s_{m}(x)} u^{(k)}(x)$, $K u(x)=\frac{\lambda_{1}}{s_{m}(x)} \int_{a}^{b} \sum_{i=0}^{p} K_{1 i}(x, t) u^{(i)}(t) d t+\frac{\lambda_{2}}{s_{m}(x)} \int_{a}^{x} \sum_{j=0}^{q} K_{2 j}(x, t) u^{(j)}(t) d t$,
Eqs (8)-(10) are the cruicial for the numerical investigations and perfomance of comparisons.

\section{METHODOLOGY}

\section{Modified Homotopy Perturbation Method}

Standard HPM for Eq. (6) is usually given by

$$
H(v, p)=(1-p) F(v)+p\left\lfloor v-h_{l}-L_{l}^{-1}\left\{-S_{l} v+f_{l}+K_{l} v\right\}\right\rfloor,
$$

where $F(v)$ is a functional operator with known solution $u_{0}$, which can easily be obtained.

The convex homotopy (11) continuously trace an implicitly defined curve from a starting point $H(u(x), 0)$ to a solution function $H(u(x), 1)$ . The embedding parameter $p$ monotonically increases from zero to unit as trivial problem $F(u)=0$ is continuously deformed to original problem $u_{l}=h_{l}+L_{l}^{-1}\left\{-S_{l} u+f_{l}+K_{l} u\right\}$. Improved HPM has been presented in Ghorbani and Saberi-Nadjafi [11]. By using this method, we define $F(v)=v-\sum_{r=0}^{N} \alpha_{r} g_{r}(x)$ where $\alpha_{r}$ are called accelerator parameters and finding these parameters under some conditions we might have exact solution or approximate solution. Let us construct MHPM as follows

$H_{l}(v, \alpha, p)=(1-p)\left(v-\sum_{r=0}^{N} \alpha_{r} g_{r}(x)\right)+p\left[v-h_{l}-L_{l}^{-1}\left(-S_{l} v+f_{l}+K_{l} v\right)\right]$,

where $\alpha_{r}$ are the parameters to be defined and $g_{r}(x)$ are given selective functions. Forcing $H_{l}(v, \alpha, p)=0$ leads to the equation

$$
v=\sum_{r=0}^{N} \alpha_{r} g_{r}(x)+p\left\lfloor h_{l}+L_{l}^{-1}\left(-S_{l} v+f_{l}+K_{l} v\right)-\sum_{r=0}^{N} \alpha_{r} g_{r}(x)\right\rfloor .
$$

Let us search approximate solution $v$ in the form of a power series

$v(x)=\sum_{n=0}^{\infty} p^{n} v_{n}(x)$

Truncated series of (14) at $p=1$ is

$$
v(x)=\sum_{k=0}^{n} v_{k}(x)
$$

Substituting (14) into (13) yields

$$
\begin{aligned}
\sum_{n=0}^{\infty} p^{n} v_{n}(x) & =\sum_{r=0}^{N} \alpha_{r} g_{r}(x) \\
& +p\left[h_{l}+L_{l}^{-1}\left(-S_{l}\left(\sum_{n=0}^{\infty} p^{n} v_{n}(x)\right)+f_{l}+K_{l}\left(\sum_{n=0}^{\infty} p^{n} v_{n}(x)\right)\right)-\sum_{r=0}^{N} \alpha_{r} g_{r}(x)\right] .
\end{aligned}
$$

Comparing the like powers of parameter $p$, we get the following schemes

$$
\begin{aligned}
& v_{0}=\sum_{r=0}^{N} \alpha_{r} g_{r}(x), \\
& v_{1}=h_{l}+L_{l}^{-1}\left(-S_{l} v_{0}+f_{l}+K_{l} v_{0}\right)-\sum_{r=0}^{N} \alpha_{r} g_{r}(x), \\
& v_{n}=L_{l}^{-1}\left(-S_{l} v_{n-1}+K_{l} v_{n-1}\right), n \geq 2 .
\end{aligned}
$$


Remark: In MHPM the accelerating parameters $\alpha_{r}$ are defined by forcing $v_{1}=0$. It leads two step iteration and gives exact solution in many cases. If $v_{1} \neq 0$ but $v_{1}^{N} \rightarrow 0$ as $N \rightarrow \infty$ then the contribution of $v_{n}, n \geq 2$ to the solution will be small therefore we can neglect $v_{n}, n \geq 2$ to find the approximate solution.

Next, for the case of $l=0$ in Eq.(15), we have the following schemes

$$
\begin{aligned}
& v_{0}=\sum_{r=0}^{N} \alpha_{r} g_{r}(x), \\
& v_{1}=h+L^{-1}\left(-S v_{0}+f+K v_{0}\right)-\sum_{r=0}^{N} \alpha_{r} g_{r}(x), \\
& v_{n}=L^{-1}\left(-S v_{n-1}+K v_{n-1}\right), n \geq 2 .
\end{aligned}
$$

The scheme (18) is used for finding exact or approximate solution of Eq. (1).

\section{Convergence and error estimation}

Let us consider the space of continuously differentiable functions $C^{m}([a, b])$ equipped with the norm

$$
\|v\|_{\infty, m}=\sum_{k=0}^{m} \max _{a \leq x \leq b}\left|v^{(k)}\right|=\sum_{k=0}^{m}\left\|v^{(k)}\right\|_{\infty},
$$

where $\Pi \Pi$ is the standard norm in $C[a, b]$.

Let the norm of kernels be defined as

$$
\begin{aligned}
& \left\|K_{1} *\right\|_{\infty}=\sum_{i=0}^{p} \max _{a \leq x, t \leq b}\left|K_{1 i}(x, t)\right|=\sum_{i=0}^{p}\left\|K_{1 i}\right\|_{\infty}, \\
& \left\|K_{2} *\right\|_{\infty}=\sum_{j=0}^{q} \max _{a \leq x, t \leq b}\left|K_{2 j}(x, t)\right|=\sum_{j=0}^{q}\left\|K_{1 j}\right\|_{\infty} .
\end{aligned}
$$

If $v_{1}=0$ in Eq. (17), then $v_{0}$ satisfies

$$
v_{0}=h_{l}+L_{l}^{-1}\left(-S_{l} v_{0}+f_{l}+K_{l} v_{0}\right)
$$

and coincides with exact solution. If $v_{1} \neq 0$, then $v_{1}^{N} \rightarrow 0$ as $N \rightarrow \infty$ . It means that for any $\varepsilon$ there exists $N_{0}$, such that $N>N_{0}$ implies

$$
\left\|v_{1}^{N}\right\|<\varepsilon
$$

Let

$$
M_{1 l}=\max _{a \leq t \leq b}\left|\frac{s_{k}(t)}{s_{m-l}(t)}\right|, M_{2 l}=\max _{a \leq t \leq b}\left|\frac{1}{s_{m-l}(t)}\right|,
$$

and

$$
\beta_{l}=\frac{(b-a)^{m-l}}{(m-l) !}\left[M_{1 l}+\left(\left|\lambda_{1}\right||| K_{1} *\left\|+\left|\lambda_{2}\right|\right\| K_{2} * \|\right) M_{2 l}(b-a)\right] .
$$

Then convergence of the approximate solution (15) is given in the following theorem.

Theorem 1: Let $K_{1 i}(x, t), K_{2 i}(x, t) \in C([a, b] \times[a, b]) \quad$ and $s_{k}(x) \in C[a, b], k=0,1, \ldots, m \quad$ with $s_{m-l}(x) \neq 0, l=\{0,1, \ldots, m-1\}, \forall x \in[a, b]$ as well as $f(x)$ be continuous function. If first iteration $\left\|v_{1}\right\|_{\infty, m}$ is bounded and $\beta_{l}$ satisfying the inequality $0<\beta_{l}<1$, and selective functions $g_{r}(x), r=0, \ldots N$ are chosen as continuous function on the interval $[a, b]$, then the series (14) with (17) is uniformly convergent to the exact solution $u(x)$ in the senses of $C^{m-l}, 0 \leq l \leq m-1$ norm on the interval $_{[a, b]}$ for each $p \in[0,1]$.

Proof. Let $\left\|\sum_{r=0}^{N} \alpha_{r} g_{r}(x)\right\|=M$ and due to (17) we obtain

$$
\begin{aligned}
\left\|v_{0}\right\|_{\infty} & =\left\|\sum_{r=0}^{N} \alpha_{r} g_{r}(x)\right\|=M, \\
\left\|v_{1}\right\|_{l, \infty} & =\left\|h_{l}+L_{l}^{-1}\left(-S_{l} v_{0}+f_{l}+K_{l} v_{0}\right)-\sum_{r=0}^{N} \alpha_{r} g_{r}(x)\right\|_{\infty} \leq \varepsilon, \\
\left\|v_{n}\right\|_{l, \infty} & =\left\|L_{l}^{-1}\left(-S_{l} v_{n-1}+K_{l} v_{n-1}\right)\right\|, \quad k \geq 2 .
\end{aligned}
$$

Let us consider the case of $n=2$. From (25) and taking into account (7) we have

$$
\begin{aligned}
\left\|v_{2}\right\|_{l, \infty} & =\left\|L_{l}^{-1}\left(-S_{l} v_{1}+K_{l} v_{1}\right)\right\|_{\infty} \\
& =\left\|\frac{1}{(m-l-1) !} \int_{a}^{x}(x-t)^{m-l-1}\left(-S_{l} v_{1}(t)+K_{l} v_{1}(t)\right) d t\right\| \leq \frac{(b-a)^{m-l}}{(m-l) !}\left\|-S_{l} v_{1}+K_{l} v_{1}\right\| \\
& =\frac{(b-a)^{m-l}}{(m-l) !} \max _{a \leq t \leq b} \mid-\sum_{k=0}^{m-l-1} \frac{s_{k}(t)}{s_{m-l}(t)} v_{1}^{(k)}(t) \\
& +\frac{\lambda_{1}}{s_{m-l}(t)} \int_{a}^{b} \sum_{i=0}^{p} K_{1 i}(t, \tau) v_{1}^{(i)}(\tau) d \tau+\frac{\lambda_{2}}{s_{m-l}(t)} \int_{a}^{t} \sum_{j=0}^{q} K_{2 j}(t, \tau) v_{1}^{(j)}(\tau) d \tau \mid
\end{aligned}
$$

Due to (23) and (24), we obtain

$\left\|v_{2}\right\|_{\infty} \leq \frac{(b-a)^{m-l}}{(m-l) !}\left[M_{1 l}+\left(\left|\lambda_{1}\right|\left\|K_{1} *\right\|+\left|\lambda_{2}\right|\left\|K_{2} *\right\|\right) M_{2 l}(b-a)\right]\left\|v_{1}\right\|_{\infty, m} \leq \beta_{l}\left\|v_{1}\right\|_{\infty, m}$,

Similarly, for $n=3$,

$\left\|v_{3}\right\|_{l, \infty}=\left\|L_{l}^{-1}\left(-S_{l} v_{2}+K_{l} v_{2}\right)\right\|_{\infty}$

$$
\begin{aligned}
& =\left\|\frac{1}{(m-l-1) !} \int_{a}^{x}(x-t)^{m-l-1}\left(-S_{l} v_{2}(t)+K_{l} v_{2}(t)\right) d t\right\| \\
& \leq\left\{\frac{(b-a)^{m-l}}{(m-l) !}\left[M_{1 l}+\left(\left|\lambda_{1}\right|\left\|K_{1} *\right\|+\mid \lambda_{2}\left\|K_{2} *\right\|\right) M_{2 l}(b-a)\right]\right\}\left\|v_{1}\right\|_{\infty, m} \leq \beta_{l}^{2}\left\|v_{1}\right\|_{\infty, m} .
\end{aligned}
$$

Continuation of this procedure yields

$$
\begin{aligned}
\left\|v_{n}\right\|_{\infty} & =\left\|L_{l}^{-1}\left(-S_{l} v_{n-1}+K_{l} v_{n-1}\right)\right\|_{\infty} \\
& \left.\leq\left\{\frac{(b-a)^{m-l}}{(m-l) !}\left[M_{1 l}+\left(\left|\lambda_{1}\right|\left\|K_{1} *\right\|+\left|\lambda_{2}\right|\left\|K_{2} *\right\|\right) M_{2 l}(b-a)\right]\right\}\right\}^{n-1}\left\|v_{1}\right\|_{\infty, m} \\
& \leq \beta_{l}^{n-1}\left\|v_{1}\right\|_{\infty, m} .
\end{aligned}
$$

The series (14) is convergent if $0<\beta_{l}<1$, therefore the series (14) at $p=1$ has the form 
$\|v\| \leq\left\|v_{0}\right\|+\sum_{n=0}^{\infty}\left\|v_{n}\right\|_{l, \infty} \leq M+\sum_{n=1}^{\infty} \beta_{l}^{n}\left\|v_{1}\right\|_{\infty, m}=M+\frac{1}{1-\beta_{l}}\left\|v_{1}\right\|_{\infty, m}$.

Since the series in (26) is geometric series with the common ratio $\beta_{l}$. Therefore, it is convergent if common ratio $0<\beta_{l}<1$. It implies that $v(x)$ is uniformly convergent on $[a, b]$.

\section{Numerical Results}

Example 1: Consider a fourth order FVIDE as

$$
\begin{aligned}
\left(e^{x}\right) u^{(4)}(x) & =\frac{3}{40} e^{x}+\frac{1}{8} e^{x+1}-\frac{1}{2} e^{x-1}-\frac{2}{15} e^{x} x^{3}-\frac{1}{10} e^{3 x} x+\frac{321}{20} e^{3 x} \\
& -\frac{1}{8} \int_{0}^{1} e^{x-t} u(t) d t+\frac{1}{5} \int_{0}^{x} t e^{x} u(t) d t, \\
u(0) & =1, u^{\prime}(0)=4, u^{\prime \prime}(0)=4, u^{\prime \prime \prime}(0)=8 .
\end{aligned}
$$

with exact solution $u(x)=e^{2 x}+2 x$.

Eq. (27) satisfied all condition in Theorem 1. We choose functions $g_{r}(x)=x^{r}$, then $v_{0}=\alpha_{0}+\alpha_{1} x+\alpha_{2} x^{2}+\cdots+\alpha_{N} x^{N}$. Therefore, we solve the algebraic equations by substituting points $x_{n}$ defined by

$$
x_{i}=\frac{i}{N}, \quad i=0,1, \ldots N
$$

\section{)}

Table 1, presents errors of MHPM for $N=\{4,6,8,10\}$ and $m=2$ of Eq. (27).

Table 1 Errors of MHPM for Eq.(27)

\begin{tabular}{cc}
\hline$N$ & $\|u-v\|$ \\
\hline 4 & $3.0568297 \times 10^{-3}$ \\
6 & $2.7136883 \times 10^{-5}$ \\
8 & $1.6190520 \times 10^{-7}$ \\
10 & $5.2707353 \times 10^{-9}$ \\
\hline
\end{tabular}

Example 2: Consider a third order FVIDE as

$\frac{4}{2+\cos (2 x)} u^{(3)}(x)=f(x)+\frac{3}{5} \int_{0}^{\pi}(x+t \sin (x)+\cos (t)) u(t) d t+\frac{1}{5} \int_{0}^{x} t \sin (x-t) u(t) d t$, $u(0)=0, \quad u^{\prime}(0)=-\frac{1}{4}, \quad u^{\prime \prime}(0)=0$,

where the function $f(x)$ is

$$
\begin{aligned}
f(x)= & \frac{1}{9}+\frac{3}{5} \pi^{2}-\frac{1}{20} \pi^{4} x+\frac{4}{5} x^{2}-\frac{1}{15} x^{4}-\left(\frac{3}{80} \pi+\frac{1}{25} \pi^{5}\right) \sin (x) \\
& -\frac{1}{60} x \sin (x) \cos (x)+\frac{29}{18} \cos (x)-\frac{1}{45} \cos ^{2}(x) .
\end{aligned}
$$

The exact solution of Eq. (29) is $u(x)=\frac{\sin (-2 x)}{8}+\frac{x^{3}}{3}$.

For this example, the inequality (24) is not satisifed in Theorem 1 . We choose selective function as $g_{r}(x)=x^{r}$ and apply the same calculations as Example 1. As a result, the errors are shown in Table 2.
Table 2 Errors of MHPM for Eq. (29)

\begin{tabular}{cc}
\hline$N$ & $\|u-v\|$ \\
\hline 4 & $2.6733302 \times 10^{-1}$ \\
6 & $1.9034554 \times 10^{-2}$ \\
8 & $9.5209585 \times 10^{-4}$ \\
10 & $3.3985516 \times 10^{-5}$ \\
\hline
\end{tabular}

From Table 2, it can be seen that even if condition of Theorem 1 is not satisfied but convergent still can be provided. This is because of neseccary condition.

Example 3: Consider a second order FVIDE as $u^{\prime \prime}(x)+x u^{\prime}(x)-x u(x)=e^{x}-2 \sin (x)+\int_{-1}^{1} \sin (x) e^{-t} d t, \quad u(0)=1, u^{\prime}(0)=1$,

The exact solution of Eq. (30) is $u(x)=e^{x}$.

Condition in Theorem 1 for the $\beta_{0}$ is not satisfied for Eq. (30). The errors of Eq. (30) for $N=\{4,6,8,10\}$ are shown in Table 3 and comparison of errors with Taylor method by Akyüz-Daşcioĝlu and

\begin{tabular}{|c|c|c|c|c|}
\hline \multirow{2}{*}{$x$} & \multicolumn{2}{|c|}{ Taylor Method [28] } & \multicolumn{2}{|c|}{ MHPM } \\
\hline & $N=5$ & $N=9$ & $N=5$ & $N=9$ \\
\hline 1 & $1.75 \times 10^{-3}$ & $3.21 \times 10^{-7}$ & $3.89 \times 10^{-5}$ & $2.92 \times 10^{-10}$ \\
\hline $\cos \left(\frac{\pi}{10}\right)$ & $1.3 \times 10^{-3}$ & $1.98 \times 10^{-7}$ & $3.95 \times 10^{-5}$ & $3.61 \times 10^{-9}$ \\
\hline $\cos \left(\frac{2 \pi}{10}\right)$ & $5.14 \times 10^{-4}$ & $4.59 \times 10^{-8}$ & $7.52 \times 10^{-5}$ & $6.60 \times 10^{-10}$ \\
\hline $\cos \left(\frac{3 \pi}{10}\right)$ & $9.32 \times 10^{-5}$ & $5.56 \times 10^{-9}$ & $2.41 \times 10^{-5}$ & $1.96 \times 10^{-10}$ \\
\hline $\cos \left(\frac{4 \pi}{10}\right)$ & $5.97 \times 10^{-6}$ & $6.28 \times 10^{-10}$ & $3.95 \times 10^{-5}$ & $1.02 \times 10^{-10}$ \\
\hline 0 & 0 & 0 & 0 & 0 \\
\hline $\cos \left(\frac{6 \pi}{10}\right)$ & $3.55 \times 10^{-6}$ & $6.23 \times 10^{-10}$ & $3.58 \times 10^{-5}$ & $1.05 \times 10^{-10}$ \\
\hline $\cos \left(\frac{7 \pi}{10}\right)$ & $2.21 \times 10^{-5}$ & $2.78 \times 10^{-9}$ & $1.86 \times 10^{-5}$ & $2.38 \times 10^{-10}$ \\
\hline $\cos \left(\frac{8 \pi}{10}\right)$ & $2.74 \times 10^{-4}$ & $2.09 \times 10^{-8}$ & $6.23 \times 10^{-5}$ & $4.27 \times 10^{-10}$ \\
\hline $\cos \left(\frac{9 \pi}{10}\right)$ & $7.89 \times 10^{-4}$ & $1.38 \times 10^{-7}$ & $3.14 \times 10^{-5}$ & $2.79 \times 10^{-10}$ \\
\hline-1 & $1.08 \times 10^{-3}$ & $2.35 \times 10^{-7}$ & $2.90 \times 10^{-5}$ & $5.53 \times 10^{-10}$ \\
\hline
\end{tabular}
Sezer [28] is shown in Table 4.

Table 3: Errors of MHPM for Eq. (30)

\begin{tabular}{cc}
\hline$N$ & $\|u-v\|$ \\
\hline 4 & $2.5349859 \times 10^{-3}$ \\
6 & $2.0592121 \times 10^{-6}$ \\
8 & $7.5200474 \times 10^{-8}$ \\
10 & $5.6622425 \times 10^{-9}$ \\
\hline
\end{tabular}

Table 4 Comparison of errors by Taylor Method [28] and MHPM for Eq.(30) 
Example 4: Consider a FVIDE as

$$
\begin{aligned}
& u^{\prime}(x)+x u(x)=f(x)+\int_{0}^{1}(1+x t) u(t) d t+\int_{0}^{x}(x-t) u(t) d t, \\
& u(0)=1 .
\end{aligned}
$$$$
\text { where } f(x)=-\sin (x)+x \cos (x)-1+\cos (x)+x-\sin (1)-x \cos (1)-x \sin (1)
$$

with exact solution $u(x)=\cos (x)$.

Errors of approximate solutions for Eq. (31) with $N=\{4,6,8,10\}$ is shown in Table 5.

Table 5 Errors of MHPM for Eq.(26)

\begin{tabular}{cc}
\hline$N$ & $\|u-v\|$ \\
\hline 4 & $1.6760159 \times 10^{-5}$ \\
6 & $3.6975135 \times 10^{-8}$ \\
8 & $4.3460143 \times 10^{-11}$ \\
10 & $1.9355063 \times 10^{-11}$ \\
\hline
\end{tabular}

Comparison of errors with Lagrange polynomial presented in Mustafa and Muhammad [23] are shown in Table 6 and 7 for $N=\{5,8\}$, respectively.

Table 6 Comparison of errors by Lagrange polynomial [23] and MHPM for $N=5$ for Eq. (31)

\begin{tabular}{ccc}
\hline$x$ & $\begin{array}{c}\text { Lagrange Polynomial } \\
{[23]}\end{array}$ & MHPM \\
\hline 0.2 & $2.392 \times 10^{-5}$ & $1.008 \times 10^{-7}$ \\
0.4 & $2.857 \times 10^{-5}$ & $2.390 \times 10^{-7}$ \\
0.6 & $3.409 \times 10^{-5}$ & $3.456 \times 10^{-7}$ \\
0.8 & $3.818 \times 10^{-5}$ & $4.971 \times 10^{-7}$ \\
1.0 & $4.300 \times 10^{-5}$ & $4.636 \times 10^{-7}$ \\
\hline
\end{tabular}

Table 7 Comparison of errors by Lagrange polynomial [23] and MHPM for Eq. (31)

\begin{tabular}{ccc}
\hline$x$ & $\begin{array}{c}\text { Lagrange Polynomial } \\
{[23]}\end{array}$ & MHPM \\
\hline 0.125 & $1.266 \times 10^{-9}$ & $2.085 \times 10^{-14}$ \\
0.25 & $1.452 \times 10^{-9}$ & $1.056 \times 10^{-12}$ \\
0.375 & $1.671 \times 10^{-9}$ & $2.683 \times 10^{-12}$ \\
0.5 & $1.867 \times 10^{-9}$ & $5.254 \times 10^{-12}$ \\
0.625 & $2.056 \times 10^{-9}$ & $8.535 \times 10^{-12}$ \\
0.75 & $2.223 \times 10^{-9}$ & $1.301 \times 10^{-11}$ \\
0.875 & $2.387 \times 10^{-9}$ & $1.777 \times 10^{-11}$ \\
1.0 & $2.490 \times 10^{-9}$ & $2.783 \times 10^{-11}$ \\
\hline
\end{tabular}

Tables 1, 2 and 3 concludes that MHPM converges to the exact solution by increasing the number of node points $x_{i}, i=0,1, . ., N$ and selective functions $g_{i}(x), i=0,1, \ldots, N$. Table 5 shows that MHPM is convergent but not uniformly to the exact solution. Tables 4,6 and 7 show the comparisons between the other methods and MHPM. It is clearly seen that MHPM gives more accurate results compare to Taylor polynomials [28] and Lagrange polynomials [23].

\section{CONCLUSION}

In this work, MHPM is used to solve FVIDE of order $m$ in general case. In MHPM was introduced accelerating parameters $\alpha=\left[\alpha_{r}\right]$ and selective functions $g(x)=\left[g(x)_{r}\right]$. The unknown parameters $\alpha=\left[\alpha_{r}\right]$ are obtained by equating $v_{1}=0$ which lead to two step approximate solution. Theorem 1 presents that MHPM for problem (1) converges uniformly when $0<\beta_{l}<1$ in the senses of norm convergence. Additionally, the numerical results exhibit that approximate solutions are still converges when condition $0<\beta_{l}<1$ does not satisfied. MHPM could avoid long and complex computations as shown in the numerical examples.

\section{ACKNOWLEDGMENT}

This work was supported by Universiti Sains Islam Malaysia (USIM) under Research Grand PPP/USG-0216/FST/30/15316. We are very grateful for sponsor and financial support of Research Management Center (RMC), USIM.

\section{REFERENCES}

[1] He J.-H, Homotopy perturbation technique, Comp. Methods Appl. Mech. Engrg., 178, 1999, pp. 257-262.

[2] He J.-H, A coupling method of a homotopy technique and a perturbation technique for non-linear problems, Int. J. Nonlinear Mech, 35, 2000, pp. 37-43.

[3] Ramos J. I., Piecewise homotopy methods for nonlinear ordinary differential equations, Appl. Math. Comput., 198, 2008, pp. 92-116.

[4] Madani M., Fathizadeh M., Khan Y., Yildrim A., On coupling of the homotopy perturbation method and Laplace transformation, Math. Comp. Modelling., 53, 2011, pp. 1937 1945.

[5] Khan Y., Wu Q., Homotopy perturbation transform method for nonlinear equations using He's polynomials, Comp. Math. Appl., 61, 2011, pp. 1963-1967.

[6] Khan Y., Faraz N, Kumar S., Yildirim A., A coupling method of homotopy perturbation and laplace transformation for fractional models, U.P.B. Sci. Bull., Series A, 74(1), 2012, pp 57-68.

[7] Ghasemi M., Kajani M. T., Davari A., Numerical solution of the nonlinear Volterra-Fredholm integral equations by using Homotopy Perturbation Method, Appl. Math. Comput., 188, 2007, pp. 446-449.

[8] Ghasemi M., Kajani M. T., Babolian E., Numerical solutions of the nonlinear integro-differential equations: Wavelet-Galerkin method and homotopy perturbation method. Applied Mathematics and Computation 188 (2007) 450-455.

[9] Eshkuvatov Z.K., Zulkarnain F.S., Nik Long N.M.A. and Muminov Z. Error Estimations of Homotopy Perturbation Method for linear Integral and Integro-Differential Equations of the Third kind, Research \& Reviews: Journal of Statistics and Mathematical Sciences. 2(1), 2016, pp. 93-101.

[10] Javidi M., Golbabai A., Modified homotopy perturbation method for solving non-linear Fredholm integral equations, Cha. Slts. Fract., 40, 2009, pp. 1408-1412.

[11] Ghorbani A, Saberi-Nadjafi J., Exact solutions for nonlinear integral equations by a modified homotopy perturbation method, Computers and Mathematics with Applications 56, 2008, pp. 1032-1039.

[12] Zaid M. Odiba, A new modification of the homotopy perturbation method for linear and nonlinear operators, Applied Mathematics and Computation, 189, 2007, pp. 746-753.

[13] Chowdhury M.S.H., Hassan T.H., Mawa S., A New Application of Homotopy Perturbation Method to the Reaction-diffusion Brusselator Model. Proc.-Soc. Beh. Sci., 8, 2010, pp. 648-653.

[14] Chowdhury M.S.H., Hashim I., Application of multistage homotopy-perturbation method for the solutions of the Chen system, Nonl. Anal.: Real Wrld. Appl., 10(1), 2009, pp. 381391.

[15] Eshkuvatov Z. K., Zulkarnain F. S., Nik Long N. M. A., Muminov Z., Modified homotopy perturbation method for solving hypersingular integral equations of the first kind, 
Springer Plus 5, 1473, 2016, 1-21.

[16] Zulkarnain F. S., Eshkuvatov Z. K., Nik Long N. M. A., Ismail F., Modified homotopy perturbation method for solving hypersingular integral equations of the second kind, AIP Conference Proceedings, 1739, 020027, 2016, doi.org/10.1063/1.4952507

[17] Brunner H., On the numerical solution of nonlinear VolterraFredholm integral equation by collocation methods, SIAM $J$. Numer. Anal., 27(4), 1990, pp. 987-1000.

[18] Hendi F. A., Albugami A. M., Numerical solution for Fredholm-Volterra integral equation of the second kind by using collocation and Galerkin methods, J. King Saud Univ. (Sci.), 22, 2010, pp. 37-40.

[19] Calió F., Muñaz M.V.F., Marchetti E., Direct and iterative methods for the numerical solution of mixed integral equations, Appl. Math. Comput., 216, 2010, pp. 3739-3746.

[20] Dastjerdi H. L., Ghaini F. M., Numerical solution of VolterraFredholm integral equations by moving least square method and Chebyshev polynomials, Appl. Math. Model., 36, 2012, pp. 3283-3288.

[21] Chen Z., Jiang W., An approximate solution for a mixed linear Volterra-Fredholm integral equation, Appl. Math. Lett., 25 , 2012, pp. 1131-1134.

[22] Bildik N., Inc M., Modified decomposition method for nonlinear Volterra-Fredholm integral equations, Cha. Slts. Fract., 33, 2007, pp. 308-313.

[23] Mustafa M. M., Muhammad A. M., Numerical Solution solution of Linear Volterra-Fredholm Integro-Differential Equations Using Lagrange Polynomials, Mathematical Theory and Modelling, 4(9), 2014, pp. 159-166.

[24] Dehghan M., Shakeri F., Solution of an integro-differential equation arising in oscillating magnetic fields using He's homotopy perturbation method. Prog Electromagn. Res. 78, 2008, pp. 361-376.

[25] Al-Hayani W., Solving $n t h$-Order Integro-Differential Equations Using the Combined Laplace Transform-Adomian Decomposition Method, Applied Mathematics, 4, 2013, pp. 882-886.

[26] Soler V., Defez E., Verdoy J. A., On Exact Series Solution for Strongly Coupled Mixed Parabolic Boundary Value Problems, Hindawi Publishing Corporation, Abstract and Applied Analysis, 2014, Article ID 759427, 9 pages

[27] Thieme H.R., A model for the spatio spread of an epidemic, $J$. Math. Biol., 4, 1977, pp. 337.

[28] Akyüz-Das cio $\hat{g}$ lu A., Sezer M., A Taylor polynomial approach for solving high-order linear Fredholm integrodifferential equations in the most general form, International Journal of Computer Mathematics, 84(4), 2007, pp. 527-539.

[29] Mohamad Nor, Md Ismailv A. I., Abdul Majid, A new homotopy function for solving nonlinear equations, AIPCP, 1557(21), 2015, pp. 21-25.

[30] Jafari H., Alipour M., Tajadodi H., Convergence of homotopy perturbation method for solving integral equations, Thai $J$. Math., 8, 2010, pp. 511-520.

[31] Kanwal R. P, Linear Integral Equations. Springer Science+Business Media, LLC. 1997. 